\title{
ENGINEERING DESIGN EDUCATION IN THERMAL SYSTEMS LABORATORY
}

Session 3226

\author{
Lang Wah Lee \\ Tamer Ceylan \\ Department of Mechanical/Industrial Engineering \\ University of Wisconsin-Platteville \\ Platteville, WI 53818
}

\begin{abstract}
This paper presents four methods to integrate design to an equipment-intensive laboratory course. These four methods are: (1) design of measurement systems with a graphical programming method; (2) design of components for testing; (3) design of experimental procedure with statistical design of experiments; and (4) evaluation of industrial products. Examples are used to illustrate the advantages and drawbacks of each method.
\end{abstract}

\section{INTRODUCTION}

To educate a new generation of engineers for the twenty-first century, engineering educators face many challenges such as the development of students' ability in critical thinking, creativity, collaborative work, and communication. Laboratory education can play a pivotal role in attaining these goals. However, due to the limitation of traditional laboratory teaching, its potential benefits have never been fully realized. The problem becomes more acute if the laboratory course is equipment-intensive. Since students are usually not familiar with the operation of equipment, they need to follow a set of detailed and rigid experimental procedures. Such a "cookbook" approach reduces students to a rather passive role in which their initiatives, creativity and motivation are suppressed. To improve the effectiveness of laboratory education, educators must develop and adopt new approaches to inspire creativity and curiosity among students. One promising approach to improve quality of laboratory education is through integration of design activities into laboratory courses.

This paper provides an account on how we integrated design education in the Thermal Systems Laboratory course. This required course is offered to senior mechanical engineering students after they have completed four prerequisite courses in Thermodynamics, Fluid Dynamics, Applied Thermodynamics, and Heat Transfer. The course is equipment-intensive. Students conduct experiments on test setups such as steam turbines, wind tunnel, centrifugal pump, refrigeration, heat exchanger, and compressor. To implement design education in this course, one needs to consider the following questions: (1) What constitutes as design activity in the lab course and how to implement it? (2) How much design content should be included in the course? (3) What type of equipment is needed? This paper will first discuss these important questions, followed by a brief description on some typical design projects and discussion on the advantage and drawbacks of various approaches. 


\section{DESIGN IN THERMAL SYSTEMS LABORATORY COURSE}

Due to the specific nature and objectives of laboratory education, implementation of design education in the course would be somewhat different from that in other courses. Educators must first clarify what constitutes as design in a lab course before the implementation. They must also decide on the appropriate amount of design content in the course.

\section{1) W hat Constitutes Design E ducation in Thermal Systems Lab}

The definition of design put forward by ABET [1] will be used as the guidance. Part of the ABET statement says "Engineering design is the process of devising a system, component, or process to meet desired needs . . . The engineering design component of a curriculum must include most of the following features: development of student creativity, use of open ended problems, development of modern design theory and methodology, formulation of design problem statements and specifications, consideration of alternative solutions ...". Educational activities in the lab that conform with the above statement would thus qualify as design work. For this equipment-intensive lab course, we have identified several approaches that would meet the ABET's design definition. The following are four of these approaches that have been tried successfully since 1996.

a) Design of Measurement Systems. We have developed a virtual laboratory component in the lab course. A state-of-the-art software program, LabVIEW, was used for developing instrument workbenches that look, operate, and produce results similar to real ones [2]. Students can use this software to build measurement systems and explore their ideas that cannot be achieved in a real setting because of cost and safety concerns.

b) Design of Components and Experimental Procedures. While it is not practical for students to design and build the entire test setup because of time and cost constraint, it is quite feasible to let the students design and build some relative simple components for testing.

c) Statistical Experimental Design. The performance of devices in thermal system is usually governed by several input parameters. Experimenters often face the challenge of finding the most efficient scheme to conduct testing and extract as much useful information as possible from a limited number of test data. The method of statistical experimental design [3] can be used by students to design a process to meet the desired needs.

d) Experimental Evaluation of Industrial System Components.

Many products from local industry would require testing to evaluate their performance. In some cases the testing is relatively simple and can serve as design projects for students.

\section{2) D esign Content}

Working on design projects would bring a lot of positive effect on the course. For example, it would shift students' role from "passive learners" to "active learners"; and change the learning 
paradigm from "learning through cookbook lab" to "learning through discovery". On the other hand, learning through design project has its own problems. It is very time consuming for both students and instructors. Too many design projects would thus limit the coverage of course material. To strike a balance between the new and traditional approaches, we found that $25 \%$ design content in the lab course is appropriate.

\section{EQUIPMENT FOR IMPLEMENTATION}

At the initial stage, the following setups and instruments in the Thermal Systems Laboratory are used for implementation of design education.

\section{1) Hardware}

- Air Conditioning and Refrigeration Education System: To conduct testing on this setup, three test parameters - flow rate of the refrigerant, the air flow rates over the condenser and the evaporator, can be varied.

- Air supply system: The system consists of two blowers, each of which can deliver $350 \mathrm{cfm}$ of air at 2 psi, and a number of differential pressure flow meters.

- Two small home-built wind tunnels: Both tunnels have the same cross sectional area (2.5" $\mathrm{X} 2.5$ ") and use the same radial-flow fan to deliver $100 \mathrm{cfm}$ of flow. Each fan is driven by a DC motor (rated at $135 \mathrm{~W}$ ). Of the two tunnels, one is used for studying both the fan laws and the flow feature at the fan outlet; the other is used for conducting forced convection heat transfer experiments. The former is equipped with two pressure transducers to measure the static fan head, a hot-wire anemometer to measure the mean air velocity and turbulence at the fan outlet, and a magnetic pickup to measure the fan rpm.

- Computers: Several Pentium computers are used for data acquisition, running the computer software (DESIGN EASE), and processing experimental data.

- Data acquisition boards: National Instrument's DAQ boards, signal conditioners, and terminal breadboards.

- Instruments: These include two pressure transducers, mounted respectively at both inlet and outlet of the fan for one of the wind tunnels, a hot wire anemometer, thermocouples, pressure differential type flow meters, and a magnetic pickup.

\section{2) Software:}

- Virtual instrument software package. National Instrument's Windows 95 LabVIEW (version 4.0.1). This software is used by students to design instrumentation systems in their design works.

- Statistical experimental design. A computer software, DESIGN-EASE [4], a product of Stat-Ease Inc., is used for the statistical experimental design.

\section{TYPICAL PROJECTS}

More than six experiments have been developed for design work in this lab. The following provides four typical examples to illustrate the four types of design activities mentioned above. 
1) Design a virtual workbench to evaluate fan performance. The LabVIEW software was used by students to design a virtual workbench. In one student design, the virtual workbench consisted of an ON/OFF switch, a speed selector to control the fan speed, and meters to record electric power consumption, fan pressure, air velocity, fan r-pm, a graph to show instantaneous air velocity, and an indicator to show the rms value of turbulent velocities. Aside from designing the virtual instrument, students also needed to consider signal conditioning and sampling. After completing the design of the measurement system, students would use it to gather data to study performance characteristics of the fan and to verify the fan laws, and to measure turbulent velocity at different fan rpms.

2) Forced convection over a flat plate. The experiment was conducted in another small wind tunnel in which a small electric plate heater is placed on the tunnel floor parallel to the flow. The convection heat transfer coefficient can be deduced by measuring the electric heat rate, the temperature of the plate, and the velocity and temperature of the air stream. Under the assumption that the heat loss from the plate to the tunnel floor is negligible, the electric heat rate is equal to the convection heat loss to the air stream. By varying the air velocity and the location of the plate, one can find the local heat transfer coefficient at various distance from the leading edge at various Reynolds numbers. The experimental results would afford a comparison with theoretical predictions. To conduct this project, students would: (a) design and construct the heated plate; (b) devise a method to insulate the plate to reduce heat loss to the tunnel floor; (c) select a method to measure the plate temperature, and (d) select an appropriate electric power to heat the plate. The experiment showed that all these four tasks must be considered carefully to ensure meaningful experimental results.

3) Performance evaluation on a refrigeration unit with statistical design of experiments technique. Students were to design a testing procedure to evaluate the effect of three parameters on the performance of vapor compression refrigeration cycle, and to search for the optimum operating condition for the unit. These three parameters are: refrigerant flow rate, and air flow rates for both the evaporator and the condenser. The project started with a preliminary design where each student team would identify the objective, select the response, determine the design type and analysis strategy, and devise an experimental matrix. After the preliminary design, the entire lab section would work together to make an experimental schedule in which the task of testing would be divided among the teams. Test results from each team would then be pooled together so that each team would have a complete set of data for analysis and draw appropriate conclusions. The computer software (DESIGN EASE) is used to facilitate the planning and data analysis.

4) Testing of pressure relief valve of oil tanks. The task was to evaluate the flow characteristics of a pressure relief valve (provided by a local industry) for oil tanks. Air was used as the test medium. The project included the preliminary design of the test setup and the piping system, the selection of instruments, calibration of equipment, and experimental testing. The test was conducted in accordance to the ASME/ANSI test code for safety and relief valves. Based on test results, students also made recommendations on modifying the design of the valve. Due to limits in resources and time, the task of building the test apparatus was omitted, and the test was conducted on a setup developed previously from a consulting work. 


\section{OBSERVATION AND EVALUATION OF THE APPROACHES}

In this section we will discuss the merit and drawbacks of the four approaches. The discussion is intended to shed some light on the process of selecting design projects.

\section{1) Design of Data Acquisition System with Virtual Instruments.}

This approach has the following characteristics:

- Effective in teaching computer data acquisition system. After he design project, students learn the fundamental knowledge and skill on how to interface with computer. Computer data acquisition system is no longer a black box to the students.

- Versatile and flexible. The approach can be applied to any setups equipped with electronic measuring sensors. The virtual front panel can easily be modified by students without safety concerns because it is software based. As a result, it is relatively low in cost. On the other hand, the approach is limited to just one type of design, i.e., the design of data acquisition systems.

\section{2) Design of Components for Testing}

Of the four approaches listed in this paper, this is probably the most comprehensive because it would encompass the activities of formulating design statements, specifications, considering alternative designs, building the components, selecting test parameters, performing the test, and writing reports.

The approach is rather time-consuming for both students and instructors. The experimental setups for this type of design should be relatively simple because of the financial constraints and the capacities in our machine shops.

\section{3) Statistical Experimental Design}

This type of design project enables students to learn a powerful applied statistical method to handle engineering testing and design problems. Since most of the experiments currently available at the Thermal System Lab are governed by multiple variables, the method can be used in a variety of experiments. Moreover, to complete the experimental matrix would require the collaborative work among student teams. This would prepare students to work in a setting similar to that in industry.

In order to implement this type of design, it is necessary to provide students with some background knowledge. This problem can be alleviated if the knowledge is covered in statistics course.

\section{4) Experimental Testing on Industrial Products}

This type of endeavor would expose students to industrial codes and prepare them for engineering practice. It would also motivate students because of its industrial realism. Through the testing, students would make recommendations to improve the design of the product and have a 
chance to interact with engineers from the sponsor companies.

Due to time constraint, this type of testing is confined to relatively simple devices.

\section{CLOSURE}

Students were very positive with these approaches. Skills learned from this course on virtual instruments and statistical experimental design were used again by some students in other design courses. From the instructor's prospective, the approaches improved the quality of laboratory education because they help nurture student creativity, raise their enthusiasm in learning, and deepen their understanding of laboratory material. At the same time, the goal of integrating design into the lab course was also achieved.

\section{REFERENCES}

1) Criteria for Accrediting Programs in Engineering in the United States, 1995-96 Accreditation Cycle, Accreditation Board for Engineering and Technology, Inc., Baltimore, MD

2) LabVIEW - Graphical Programming, Gary Johnson, McGraw Hill, 1994

3) Understanding Industrial Designed Experiments, S.R. Schmidt, and R.G. Launsby, 4th Edition, Air Academic Press, 1994

4) DESIGN-EASE Software Manual, Stat-Ease Inc., Minneapolis, MN

\section{BIOGRAPHICAL SKETCHES}

\section{LANG WAH LEE}

Dr. Lee is a professor of mechanical engineering at the University of Wisconsin - Platteville. He received his M.S. Degree (1972) from California Institute of Technology and Ph.D. Degree (1975) from the University of Wyoming. Address: 1 University Plaza, Platteville, WI. 53818. Phone: (608) 342-1437. e-mail:Lee@uwplatt.edu.

\section{TAMER CEYLAN}

Dr. Ceylan is the Chair of the Mechanical/Industrial Engineering Department and a professor of mechanical engineering at the University of Wisconsin-Platteville. He received his M.S. (1976) and Ph.D. (1979) Degrees in mechanical engineering from the University of Wisconsin-Madison. Address: 1 University Plaza, Platteville, WI. 53818. Phone: (608) 342-1367. e-mail: Ceylan@uwplatt.edu. 